\title{
Seguir la revolución: las redes del movimiento LGBT+ mexicano en Twitter*
}

\author{
Continuing the revolution: the mexican LGBT+ movement's \\ networks on Twitter
}
Seguir a revolução: as redes do movimento LGBT+ mexicano no Twitter

Raúl Anthony Olmedo Neri**

\section{RESUMEN}

El uso de Internet por parte de los movimientos sociales ad-

Palabras clave: quiere relevancia en la actualidad, por lo que analizar estas maactivismo, apropianifestaciones desde la comunicación abre camino a pensar las ción, hashtag, moformas de interacción desarrollas en Twitter. A partir de ello, el vimiento LGBT+, presente trabajo realiza un análisis respecto del uso que le da Twitter. el movimiento LGBT+ mexicano a Twitter, particularmente las arquitecturas reticulares que se forman alrededor del hashtag \#Orgullo2021Mx. Con el análisis de redes sociales (ARS) se han obtenido las interacciones de diferentes usuarios con esa etiqueta, para después visualizar dichos enlaces en la red formada a través del contenido. Finalmente, entre los resultados obtenidos se encuentra que quienes participan en la red son integrantes del movimiento LGBT+ y le dan sentido a un discurso que se vincula con los significados concatenados al hashtag \#Orgullo2021Mx.
ABSTRACT
Social movements' use of the Internet is becoming relevant Keywords: acti- nowadays, so analyzing these manifestations from the commu- nication point of view opens the way to think about the forms of vism, appropria- tion, hashtag,

\footnotetext{
* Este artículo es parte del trabajo desarrollado en la actual investigación doctoral del autor, la cual recibe financiamiento del Consejo Nacional de Ciencia y Tecnología (CONACYT).

** Mexicano. Maestro en Comunicación por la Universidad Nacional Autónoma de México (UNAM). Profesor de Asignatura en la Facultad de Ciencias Políticas y Sociales de la UNAM. Ciudad de México, México.raul.olmedo@politicas.unam.mx
} 
interaction developed on Twitter. This paper examines how the Mexican LGBT+ movement uses Twitter, particularly the reticular architectures formed around the hashtag \#Orgullo2021Mx. With social media analytics (SMA), we obtained different user interactions with that tag to visualize those links in the network formed through the content. Finally, among our results, we find that those participating in the network are members of the LGBT+ movement and give meaning to a discourse linked to the meanings of the hashtag \#Orgullo2021Mx.

\section{RESUMO}

O uso da internet por parte dos movimentos sociais adquire relevância na atualidade, motivo pelo qual analisar estas manifestações desde a comunicação abre caminho para pensar nas formas de interação implementadas no Twitter. A partir disso, o presente trabalho realiza uma análise com relação ao uso do Twitter pelo movimento LGBT+ mexicano, particularmente das arquiteturas reticulares formadas em torno da hashtag \#Orgullo2021Mx. Com a análise das redes sociais (ARS) foram obtidas as interações de diferentes usuários com essa etiqueta, para depois visualizar tais vínculos na rede formada através do conteúdo. Finalmente, entre os resultados obtidos se observa que aquelas pessoas que participam desta rede são integrantes do movimento LGBT+ e dão sentido a um discurso que se vincula com os significados concatenados à hashtag \#Orgullo2021Mx.
LGBT+ movement, Twitter.

Palavras-chave: ativismo, apropriação, hashtag, movimento LGBT + , Twitter. 


\section{Introducción}

Los movimientos sociales contemporáneos reconocen el papel estratégico de la comunicación y la tecnología dentro de sus repertorios de acción. Actualmente, esta dimensión adquiere un peso estratégico y articulador que se sustenta en la arquitectura reticular de la red; por tanto, dicha dimensión muestra su relevancia no solo en el fortalecimiento de las redes internas de los movimientos, sino en la expansión de sus demandas desde el punto de vista comunicativo e informacional.

De esta manera, el ámbito comunicativo consolida su relevancia a través de los dispositivos tecnológicos e Internet, los cuales se despliegan de forma sutil y funcional en la vida cotidiana. Cuando la tecnología se vuelve invisible en la sociedad, es porque su incorporación ha derivado en un proceso que ya no se piensa más sin ese elemento articulador de sentido (Pisani y Piotet, 2009).

El movimiento Lésbico, Gay, Bisexual y Trans (en adelante LGBT+) es un ejemplo que muestra los resultados de la apropiación de la tecnología, así como el fortalecimiento de los vínculos que dotan esta acción colectiva de un carácter reticular y ubicuo (Olmedo, 2021). Cabe destacar que, aunque diferentes en cuanto a demandas y logros, todas estas poblaciones tienen como común denominador el estigma construido desde el heterosexismo, por lo que su aglutinación no deriva solo de la disidencia sexual e identitaria que representan, sino también por las transgresiones que simbolizan para la vida cotidiana construida desde la heteronorma.

Por otro lado, es menester reconocer que este movimiento no está integrado solamente por activistas y organizaciones sociales. Por el contrario, su comunidad, la población simpatizante con la agenda LGBT+, aquellos proyectos mediáticos que atienden las necesidades informativas de estas poblaciones y los espacios de socialización construidos colectivamente constituyen tanto el movimiento como lo que Enguix (2016) define como el "ambiente" LGBT+. Estos sujetos y espacios desarrollan acciones cotidianas que también contribuyen al cambio social desde la expresión cultural e identitaria de las poblaciones LGBT+. Estas acciones configuran un activismo soft, es decir, acciones indirectas de resistencia y reivindicación que muestran estas preferencias sexuales e identidades de género en espacios públicos y cotidianos. Así, el activismo soft genera rupturas en el heterosexismo y cuestiona la cultura que sustenta el estigma. 
En este sentido, la pregunta de investigación fue ¿cuáles son las implicaciones de la interacción social mediada por una etiqueta o hashtag generado por el movimiento LGBT+ mexicano? Y de manera particular, ¿de qué manera la estructura reticular de Internet permite ampliar el contenido generado por y para miembros del movimiento LGBT+? Para atender estas preguntas de carácter exploratorio, el presente trabajo analiza las estructuras reticulares que se forman a través de la generación de un hashtag en Twitter, con la finalidad de analizar las implicaciones comunicativas en las acciones del movimiento LGBT+ mexicano. Desde esta investigación, la creación y difusión de una etiqueta no solo responde a la innovación, sino a la apropiación de las lógicas operativas de la plataforma sociodigital para darle un uso creativo y atender fines específicos, en este caso de un movimiento social.

La hipótesis manejada en esta investigación es que la generación y difusión de un hashtag en Twitter desarrolla estructuras reticulares que contribuyen al flujo de contenido alternativo entre los perfiles de aquellas personas, instituciones y organizaciones que forman parte del movimiento LGBT+ mexicano. Esta hipótesis responde tanto al paradigma de la red (Rovira, 2012) como de las afinidades estructurales que posee este movimientos social e Internet.

Con el paradigma de la red aplicado a los movimientos sociales han surgido conceptos que atienden el carácter reticular de la acción colectiva. Por ejemplo, el concepto de repertorio de acción conectiva (Reguillo, 2017), permite reconocer la visibilidad de las demandas y la intervención comunicativa en la esfera pública mediante Internet. Los repertorios de acción conectiva son estrategias sinérgicas que adoptan los movimientos sociales para incrementar la visibilidad de sus acciones en las redes y en las calles: son formas convergentes de acción que amplifican las demandas, los discursos y las representaciones sociales que cuestionan los agravios por los que se movilizan. Siguiendo la propuesta conceptual de Reguillo (2017), es necesario aclarar que los repertorios de acción conectiva no adquieren este adjetivo exclusivamente por el carácter tecnológico y digital, sino por su contribución al incremento de la capacidad reticular e informativa de los participantes en y con la movilización, sin que estén condicionados por afiliaciones de orden político o sindical.

Para atender el objetivo de esta investigación se empleó el método de análisis de redes sociales (ARS), con la finalidad de recuperar 
aquellas redes que se desarrollaron alrededor de la etiqueta \#Orgullo2021Mx, la cual se empleó durante el mes de junio de 2021, mes en el que se incrementan las actividades de visibilidad y movilización social de las comunidades LGBT+ en México.

La relevancia de este análisis radica en que es la segunda ocasión en la que las diferentes marchas del Orgullo en México - entre ellas la realizada en la capital del país-, cobraron un matiz convergente, ya que se tomaron las calles y las plataformas coordinadamente, debido al contexto pandémico por COVID-19 ${ }^{1}$. Aunque este proceso de apropiación e incorporación venía desarrollándose desde inicios del siglo XXI, esta situación contextual vino a redimensionar la potencialidad de Internet para replicar, ampliar y fortalecer el carácter conectivo tanto de los movimientos sociales como de la sociedad contemporánea.

En este orden de ideas, el trabajo articula un marco teórico alrededor del diálogo entre los movimientos sociales e Internet, haciendo énfasis en el caso LGBT+ mexicano, para después presentar la metodología del ARS como potencial camino para analizar y representar visualmente las prácticas sociales en el espacio digital. Finalmente, se discuten los resultados del análisis de este marco teórico-metodológico, con la finalidad de abonar a los procesos empíricos que desarrollan en y por la comunidad LGBT+.

\section{Marco teórico}

La gran mayoría de los estudios en torno a los movimientos sociales se ha desarrollado a partir del campo sociológico. Flores-Márquez (2017) identifica tres campos generales que se han constituido desde esta vertiente: los estudios centrados en los objetivos políticos, aquellos enfocados en el ámbito económico y otros más anclados en la perspectiva cultural de las acciones colectivas. Junto con estos campos se identifica la teoría de movilización de recursos (TRM) y la teoría de la acción racional (TAR) como perspectivas que atienden el carácter organizativo

1 Cabe mencionar que hubo pequeños contingentes que realizaron los históricos recorridos en las diferentes ciudades mexicanas, aunque su impacto estuvo determinado por las restricciones sanitarias implementadas y por la infraestructura y programación que se dio en diferentes medios de comunicación respecto de la marcha. Eventos culturales transmitidos por Youtube y streaming a través de Facebook fueron formas en las que el uso de Internet sirvió de soporte para sortear las restricciones sociales de la pandemia. 
y las finalidades del movimiento social (Candón Mena, 2011). Por su parte se encuentra la teoría de los nuevos movimientos sociales (NMS), que hace énfasis en las motivaciones socioculturales e identitarias que articulan aquellos movimientos sociales presenten en los años sesenta del siglo pasado (Melucci, 2010; Touraine, 2005).

Aunque esta última perspectiva se consolida en el ámbito teórico por el reconocimiento de carácter cultural y comunicativo que presentan las acciones colectivas, lo cierto es que dichas posturas asumen de manera superficial e instrumental el carácter detonador de la comunicación en tanto proceso social (Craig, 1999). Esto diezma el alcance comunicacional que despliegan los movimientos en su devenir histórico para cumplir con sus objetivos a lo largo del tiempo.

Así, a pesar de que en la sociología existen análisis que reconocen la relevancia del campo comunicativo, particularmente con la aparición de Internet y las plataformas sociodigitales (McAdam et al., 2004; Tilly y Wood, 2010), existe una amplia literatura (Candón Mena, 2011; 2019; Mattoni y Treré, 2014; Olmedo, 2020; Rovira, 2012; Sola-Morales y Sabariego, 2020; Treré, 2020) que evidencia la superficialidad de dichos acercamientos por reducir el carácter tecnológico a una función pragmática, dejando de lado las complejas formas de desarrollar prácticas sociales en Internet y de no asumir las implicaciones tecnosociales de su apropiación por parte de las acciones colectivas.

Así, pensar los movimientos sociales desde la comunicación permite reconocer su carácter informativo, de transformación sociocultural y de articulación simbólica en el contexto convergente. De hecho, la perspectiva comunicativa de los movimientos sociales obliga a reconocer un cambio más amplio en la sociedad donde se manifiestan: el desarrollo tecnológico abre camino para proponer el concepto de sociedad beta. Esta propuesta conceptual descansa en la definición clásica de beta, la cual "se usa tradicionalmente para describir un software que funciona, pero que aún está probándose, sin acabar" (Pisani y Piotet, 2009, p. 76). Entonces, la sociedad beta reconoce el constante proceso de actualización tecnosocial que no parece tener un final, dado que la tecnología en su permanente innovación obliga a los individuos a renovar los dispositivos y las formas de incorporarlos en la vida cotidiana: se aprende y trabaja sobre la marcha, incorporando las innovaciones y repensando las prácticas ya establecidas. 
La sociedad beta está compuesta por procesos, es decir, un conjunto de fases que aún no culminan en un producto final. Este carácter en permanente apertura no se limita a sus fines, sino que se extiende a sus medios y formas de comunicación. Por tanto, la constante apropiación de la tecnología hace que los movimientos sociales estén innovando sus usos y estrategias y que construyan con ello un conocimiento a partir de la experiencia sobre estas actualizaciones en el campo de la comunicación (Olmedo, 2021).

Desde esta mirada es posible reconocer conceptos para dimensionar las acciones colectivas desde la comunicación: a nivel grupal, la propuesta de movimientos comunicacionales de Barranquero (2012) resulta útil por la intención general de nombrarlos desde este carácter comunicativo; por su parte, el término videoactivismo (Sierra y Montero, 2016) da cuenta de la vinculación entre la producción de contenido como estrategia, evidencia y memoria de los procesos de resistencia y movilización, mostrando la relación entre los movimientos sociales y el flujo de contenido dentro de Internet, así como las formas y estrategias que se configuran dentro del conflicto que emana de la resistencia de estos grupos organizados ante un agravio.

A nivel individual, Pisani y Piotet (2009) proponen el concepto de webactores, dado el carácter individual que supone la relación del sujeto y la tecnología, resaltando las posibilidades conectivas y de producción de prácticas que amplifican los canales de comunicación en la sociedad contemporánea.

Finalmente, en un nivel más general, es decir, que incluye los contextos, acciones, finalidades y resultados de los movimientos sociales se han generado propuestas conceptuales como la tecnopolítica (Rodríguez, 2020) y la comunicación emergente (Olmedo, 2020). La tecnopolítica, por ejemplo, es definida como "la capacidad que tienen los individuos y organizaciones de apropiarse de las herramientas digitales para la organización" (Burgos, 2015, p. 3). Este concepto vuelto disciplina se centra en mayor medida en la dimensión política de su uso (Sola-Morales y Sabariego, 2020), por lo que la visibilidad de su empleo radica en buena medida en estudios centrados en la clara disputa de poder entre los movimientos y quienes detentan un privilegio dentro de la vida cotidiana, sea este material, o bien, simbólico. 
En otro tenor, la comunicación emergente vincula estos procesos de comunic-acción a la trayectoria teórico-conceptual de la comunicación alternativa y la apropiación social de la tecnología. Lo anterior debido a que el uso que le dan los movimientos sociales contemporáneos a Internet y a las plataformas sociodigitales tiene la intención de intervenir en el espacio público mediante el giro comunicacional sobre el contenido, los discursos y la forma de ser/estar en el mundo. Además, este concepto reconoce la dimensión técnica, mediática, económica y social que se desprende de la apropiación de la tecnología por parte de estas acciones colectivas (Olmedo, 2020).

Aunque estos acercamientos permiten identificar el carácter positivo de esta convergencia, también reconocen las limitantes que se encuentran detrás de su uso, particularmente los intereses de los $s e$ ñores de la red (Zallo, 2016). Efectivamente, las plataformas sociodigitales encarnan procesos económicos de acumulación (Fuchs, 2015; 2021), los cuales suponen nuevas formas de explotación (Han, 2018). Estas dinámicas de subsunción de la interacción social al capital desarrollan nuevos nichos de mercado y novedosos campos de conflicto derivados de las falacias que se articulan en Internet (García y Olmedo, 2019).

De esta manera, el reconocimiento de las potencialidades de Internet para los movimientos sociales también implica avizorar aquellos intereses que sustentan en buena medida la también llamada red de redes.

Si se aplica este acercamiento comunicacional al movimiento LGBT+ se puede identificar que no existe un concepto específico que dé cuenta de las formas de incorporación de la tecnología por parte de estas poblaciones. Esto sí ocurre con el feminismo (Romero, 2014), mediante conceptos como ciberfeminismo (Rodríguez, 2020) o tecnofeminismo (Wajcman, 2006). Por ello en este trabajo se retoma la comunicación emergente como un concepto útil para dimensionar el carácter comunicativo del movimiento LGBT+ en Internet, ya que su uso "no solo permite amplificar el alcance del individuo en el proceso de comunicación, sino que posibilita la intervención en la esfera pública" (Olmedo, 2020, p. 96).

Si bien es cierto que el movimiento LGBT + ha construido una larga trayectoria de intervención y vinculación con la esfera política para dar 
atención a su agenda, lo cierto es que existe una dimensión de intervención en el espacio público mediante el activismo y la visibilidad de las comunidades: su presencia en el espacio público genera boquetes en los discursos que sustentan su discriminación.

De allí la importancia de analizar este movimiento desde la comunicación emergente, ya que esta perspectiva evidencia su carácter comunicativo, mismo que se encuentra encadenado a la vida cotidiana de sus integrantes: el reconocimiento de la disidencia sexual en el espacio público y privado ya es, por sí mismo, un mensaje dotado de un significado que en su difusión entra en conflicto con los cánones que sustentan el heterosexismo en la sociedad. Además, las afinidades culturales e identitarias que presentan sus integrantes se vuelven articuladoras de redes sociales que se replican y amplían en Internet y en las plataformas sociodigitales. Por ende, la estructura de este movimiento reafirma su carácter reticular, facilitando la apropiación de Internet al compartir esta condición estructural (Olmedo, 2021).

De hecho, este movimiento evidencia que su desarrollo adquiere matices por el contexto político, social y cultural dentro de cada país, no obstante, en ellos está presente la transversalidad de Internet en sus acciones. El estudio multisituado que realiza Martel (2013) alrededor del activismo lésbico y gay en diferentes países permite entender cómo es que se da la apropiación estratégica de la tecnología, por un lado, y de qué manera esos usos permiten sortear la discriminación emanada de la sociedad o del Estado.

Lo anterior es importante dado que aún en Internet se desarrollan prácticas de discriminación que encarnan la LGBTfobia institucional y sociocultural (Albury, 2018; Olmedo, 2020), por lo que el uso de Internet no debe entenderse en su sentido exclusivamente progresista, sino como el escenario donde confluyen los discursos hegemónicos y alternativos. Esta es otra razón que fundamenta de manera directa la apropiación de estas innovaciones tecnológicas: estar presentes en el espacio digital no solo fortalece la visibilidad de la diversidad sexual, sino que también hace frente a los discursos de odio que se articulan en el mundo virtual por parte de organizaciones de corte religioso y conservador.

En el caso mexicano, la búsqueda y gestión de espacios y medios de comunicación por parte del movimiento LGBT+ se puede encontrar en 
el siglo XX. La importancia de hacer frente a las representaciones sociales de la diversidad sexual creadas y difundidas por los medios de comunicación masiva evidencia, quizás, uno de los más grandes objetivos de este movimiento: la demanda de reconocimiento y el desmantelamiento del estigma que se ha desarrollado en la sociedad mexicana (Medina, 2015; Olmedo, 2019). Son los medios de comunicación masiva los que han contribuido a la estigmatización de las comunidades LGBT+ en México: mediante sus contenidos han construido una representación social de la diversidad sexual alienada de cualquier derecho o posibilidad de existencia. Por ello la búsqueda de medios alternativos de visibilidad y acciones de presentación en el espacio público por parte de las comunidades LGBT+ han significado los primeros procesos de resistencia ante la calumnia convertida en entretenimiento ${ }^{2}$.

Aunque las marchas del Orgullo iniciadas en la capital del país en 1978 (Díez, 2011; 2018; Lizárraga, 2018) son la más clara estrategia de visibilidad y movilización, también es importante mencionar que el movimiento LGBT+ mexicano entendió desde sus inicios que los medios de comunicación y sus contenidos eran parte del agravio que motivaba su organización y lucha. Por ello, los proyectos de revistas independientes como Nuestro Cuerpo (Simonetto, 2017), películas como El lugar sin límites de Arturo Ripstein (1977), novelas como El Vampiro de la Colonia Roma de Luis Zapata (1979), obras de teatro, la difusión y adhesión a escritos clásicos como Contra la práctica del ciudadano como botín policiaco de Luis González de Alba en 1975 (Castillo, 2016), el autorreconocimiento de Nancy Cárdenas (1934-1994) como lesbiana en el noticiero "24 horas" de Jacobo Zabludovsky en 1973 (Irys, 2018), así como el desarrollo de espacios para la reunión y divulgación de información para la comunidad LGBT+ como "Los martes de El Taller", que inició el 14 de abril de 1987 bajo el contexto del inicio del VIH-Sida en México y cuyas actividades siguen vigentes en la actualidad (Olmedo, 2020), son solo algunos ejemplos de cómo las comunidades LGBT+ mexicanas han ideado y empleado una serie de estrategias en el ámbito de la comuni-

2 La revista Alarma se caracterizó en el siglo XX como un medio que aprovechaba la homosexualidad para convertirla en un espectáculo. Muchos de sus números tuvieron en primera plana la degradación de hombres homosexuales que eran aprehendidos por la policía en las razzias, redadas realizadas en la Ciudad de México y otros estados por policías en centros nocturnos, y cuya libertad estaba condicionada a fotografías humillantes o sobornos para no delatar su orientación sexual a sus familiares. 
cación para hacer frente a los códigos simbólicos de exclusión que se manifiestan en los contenidos informativos y comerciales difundidos.

Estos antecedentes muestran el proceso de gestión y apropiación de los medios de comunicación para el desarrollo de contenido que visibiliza la diversidad sexual y cuestiona los discursos hegemónicos, en este caso articulados alrededor de las heteronormas y el estigma. Por ello el uso de Internet forma parte de este proceso histórico en el que la tecnología y la comunicación han servido para contrarrestar los prejuicios en torno a las comunidades LGBT+.

Con la llegada de Internet y las plataformas sociodigitales, el movimiento LGBT+ ha podido desarrollar un social affordance (Bucher y Heldmond, 2018), con el cual ha ampliado su frontera de acción y comunicación. Como ya se ha mencionado, en Internet y particularmente en las plataformas sociodigitales se materializa una dinámica relacional (Pisani y Piotet, 2009). En esta dinámica, las personas no solo tienen la posibilidad de replicar y ampliar sus redes sociales, sino que los vínculos también pueden estar determinados por el contenido que allí se crea y difunde. Por tanto, la capacidad de los individuos para establecer relaciones con activistas, organizaciones y medios alternativos permite reconocer en Internet y las plataformas sociodigitales la posibilidad de reducir costos de movilización e incrementar el diálogo de saberes y resistencias entre quienes integran estas comunidades, para la mejora de sus estrategias y el fortalecimiento de su trabajo de acuerdo con sus intereses (Ciszek, 2017).

Para que los vínculos y el flujo de información puedan establecerse en las plataformas sociodigitales se requiere de una condición estructural fundamental: un perfil. Este elemento es tanto el producto de la digitalización del individuo que ingresa a la plataforma como el punto desde el cual se establece la conexión con y en la red. En términos de redes el perfil sería un nodo, es decir, la unidad mínima que forma parte de una arquitectura reticular.

La relevancia del perfil yace en su capacidad conceptual de ser un lugar de enunciación; esto es factible porque desde allí el individuo establece los procesos de visibilidad y socialización en cada una de las plataformas donde interactúa. Otros autores apuestan más por entender el perfil como una plaza pública digital, ya que establece "el piso reticular 
no físico que facilita la unión entre perfiles humanos (la estructura que se forma entre las aristas y los nodos que se materializan y visibilizan en los estudios sociométricos)" (González y Servín, 2017, p. 65).

No obstante, definir el perfil digital como lugar de enunciación dimensiona el papel del sujeto dentro de esa gran malla de significados, flujos e información en la que indirectamente es producto y directamente productor (Olmedo, 2021). En otras palabras, el lugar de enunciación expresa las condiciones de clase, biográficas, de consumo y de experiencia del sujeto, todas ellas ejerciendo influencia sobre el diseño del perfil, el contenido que difunde y la interpretación que le da a los flujos de información que están en Internet.

Esto es parte sustancial de la conexión y de las redes que allí se forman, por lo que el perfil se convierte en la puerta de acceso, pero también es un elemento constitutivo del individuo que diseña de acuerdo con su experiencia biográfica. Esto le permite compartir ideas, contenidos y significados con otros perfiles, derivando en un vínculo que puede fortalecerse o desaparecer con el paso del tiempo y la interacción. Por tanto, el perfil digital no solo debe ser considerado como un lugar desde el cual se interactúa, sino que también es un espacio construido desde el posicionamiento individual que es expuesto en el espacio público; en él es posible encontrar elementos que forman parte de la identidad del individuo y que en otros lugares estarían restringidos por su transgresión a los parámetros culturales previamente establecidos o por la violencia de la que podría ser objeto. En otras palabras, el perfil también es un lugar que se diseña por elementos que forman parte de la intimidad, individualidad y colectividad de quien lo posee y utiliza.

A partir de ello es que las estructuras reticulares pueden formarse tanto por la consolidación de vínculos permanentes mediante las interfaces ${ }^{3}$ como por el contenido que es compartido, comentado y reaccionado en dichas plataformas. Pensar y actuar en red responde al cambio sustantivo que se reconoce en la sociedad desde la segunda

3 En Facebook, por ejemplo, la relación existente se da a través de la amistad entre dos o más usuarios. Aunque también existe la posibilidad de seguir/ser seguido, la amistad es el vínculo que totaliza las relaciones sociales en esta plataforma. En su caso, Twitter consolida la relación seguir/ser seguido para administrar el flujo de información. En ambos casos hay procesos de interacción mediante reacciones o intervención a partir de comentar dicho contenido. 
mitad del siglo XX. Deleuze y Guattari (2010) fueron los primeros en avizorar que la estructura arbórea para la producción de conocimiento estaba cambiando a una forma de arquitectura rizomática, donde las jerarquías se diluyen en una permanente red cuyos límites están determinados por sus componentes: nodos y enlaces.

Ese cambio estructural ha ido replicándose a otros ámbitos, de allí que asumir la noción de red permite consolidar una perspectiva propia del contexto de la sociedad actual. Las redes facilitan la difusión de información, posibilitan una redistribución del trabajo y generan un contexto permanente para el trabajo conjunto sin perder la autonomía de las organizaciones, activistas y personas que día a día avanzan hacia la idea de mundo que los motiva a tejer la revolución. Para poder establecer un acercamiento a estas redes de interacción-comunicación (Olmedo, 2020; 2021) se recurrió al análisis del uso dado a la etiqueta \#Orgullo2021Mx.

De esta manera, la factibilidad de aplicar el análisis de redes sociales (ARS) en el uso de Twitter por el movimiento LGBT+, hace posible identificar el carácter reticular que se manifiesta mediante los perfiles y el contenido.

\section{Metodología}

Con la finalidad de reconocer el carácter relacional que se gesta alrededor del hashtag \#Orgullo2021Mx se ha recurrido al ARS. Mediante el software NodeXL se recolectaron las interacciones entre usuarios de Twitter alrededor de esta etiqueta; la visualización de las redes se hizo a través del software Gephi.

Con ambos software es posible calcular diferentes indicadores propios de la estructura externa e interna de la red; para esta investigación solo se enfocó en el grado de intermediación, el cual permite entender qué nodos tienen la capacidad de distribuir o cerrar los flujos de información de acuerdo con sus propios intereses.

La selección del análisis en Twitter descansa en el acceso a los datos mediante la Application Programming Interface (API), en la relevancia de esta plataforma como espacio de generación de contenido y discusión alrededor de potenciales temas tendencia (Trending Topics), así como su posición en cuanto a la cantidad de usuarios en México 
que la utiliza (Ramón, 2021). Así, esta plataforma se consolida como una de relevancia en el contexto nacional y latinoamericano ${ }^{4}$.

Dado que el interés de la investigación se desarrolla alrededor de las interacciones de los perfiles a través del contenido que fluye en Twitter, el ARS se fortalece con el uso de la minería de datos (Gunter, 2014); la incursión de los software tiene que ver tanto por la gran cantidad de datos que se generan en Internet como por su apoyo para visualizar las redes que allí se generan. Para utilizar el ARS en las interacciones sociales digitales en Internet, es necesario reconocer: 1) la variable de trazabilidad; 2) la delimitación temporal de recolección; y 3) la delimitación en cuanto a la direccionalidad de los vínculos.

Respecto de la variable de trazabilidad, el hashtag \#OrgulloMx2021 permite rastrear su uso y con ello los perfiles que interactuaron a través de su uso o generación de contenido sobre la publicación con dicha etiqueta. Por su parte, el periodo de recolección es fundamental porque permite reconocer la contextualización del hashtag y el comportamiento de su uso en Twitter (Olmedo, 2020): cuando el hashtag encuadra con una actividad, evento o movilización en el espacio público, es posible que este incremente su actividad, visibilidad y viralidad. En otras palabras, la vinculación entre contexto y etiqueta responde a una cuestión estratégica y situada a los fines del movimiento social en cuestión.

En este caso, el periodo de recolección de información se dio durante el mes de junio de 2021, dado que en ese periodo se incrementan por ser considerado como el mes del Orgullo en México. Marchas, conferencias, talleres, seminarios, entre otros, son ejemplos de acciones que generan y difunden información de este movimiento en diferentes partes del país, por lo que el uso de ese hashtag dentro de la difusión de algún evento o acción permite obtener las huellas digitales que genera cada perfil mediante la interacción.

Finalmente, respecto de la direccionalidad de los vínculos, es posible reconocer no solo aquellos nodos que poseen un mayor número de

4 Cabe mencionar que existe una geopolítica mundial dado que detrás de cada plataforma se encuentran intereses que dan sentido a las tensiones políticas y económicas que rodean la sociedad contemporánea. Ren Ren, WeChat, QZone, Facebook, Twitter, VKontakte son ejemplos de la división global del entretenimiento y contenido a través de las plataformas en Internet. 
enlaces, sino su posición estratégica dentro del flujo de información. De esta manera, y a partir de los intereses de este trabajo, los vínculos que se han omitido son aquellos de carácter reflexivo; este tipo de vínculos se caracteriza por ser enlaces cuyo origen y destino es el mismo nodo, esto es, no tiene un carácter eminentemente conectivo. A partir de estos criterios de selección y delimitación se construyó una base datos que aglutina aquellos nodos y vínculos propios de una red en la red.

\section{Resultados}

Junto a \#Orgullo2021Mx existieron otras etiquetas como \#Matrimoniolgualitario, \#ResistenciaYUnidad y \#AmorEsAmor. Las lógicas conectivas que yacen en cada una de esas etiquetas permiten realizar un análisis particular o en su caso a través de su interrelación. Las interacciones alrededor de este hashtag encuentran una lógica con las actividades que se desarrollan durante el mes del Orgullo en México.

En la Figura 1 se evidencian las interacciones que se desarrollaron alrededor de esta etiqueta durante junio de 2021.

Figura 1

Interacciones con el hashtag \#Orgullo2021Mx en junio 2021

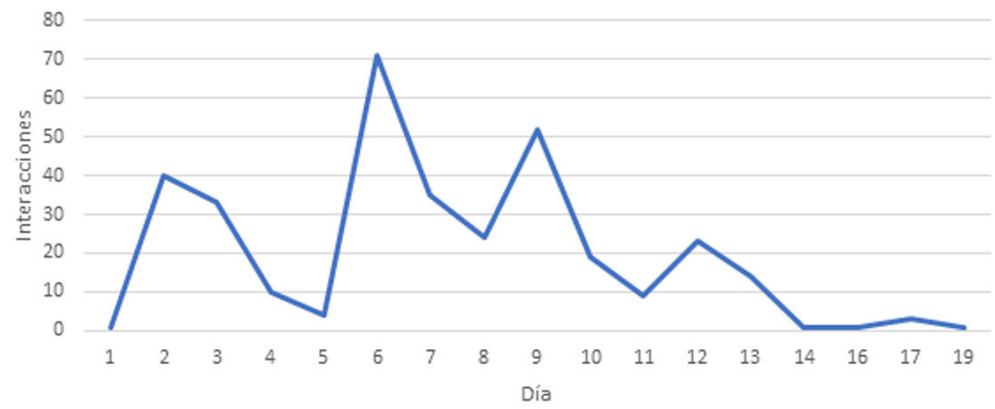

Fuente: Elaboración con datos de NodeXL.

Aunque su uso no llega a tener interacciones dentro de la última semana de junio, sí muestra que una etiqueta por sí misma no permanecerá a menos que haya un empuje en cuanto a su difusión y uso. En el caso del hashtag \#Orgullo2021Mx se identificaron 94 nodos y 341 vínculos; lo anterior muestra que 94 perfiles interactuaron 341 veces 
con dicha etiqueta durante junio. Dichas interacciones son a través del tiempo, por lo que un perfil pudo haber interactuado con esta etiqueta más de una vez.

En su conjunto, estos vínculos son lo que fundamentan la reticularidad de dicha estructura. Cada interacción da paso a un vínculo mediado, en este caso, por la etiqueta \#Orgullo2021Mx, como muestra la Figura 2.

Figura 2

Red de usuarios alrededor de \#Orgullo2021Mx

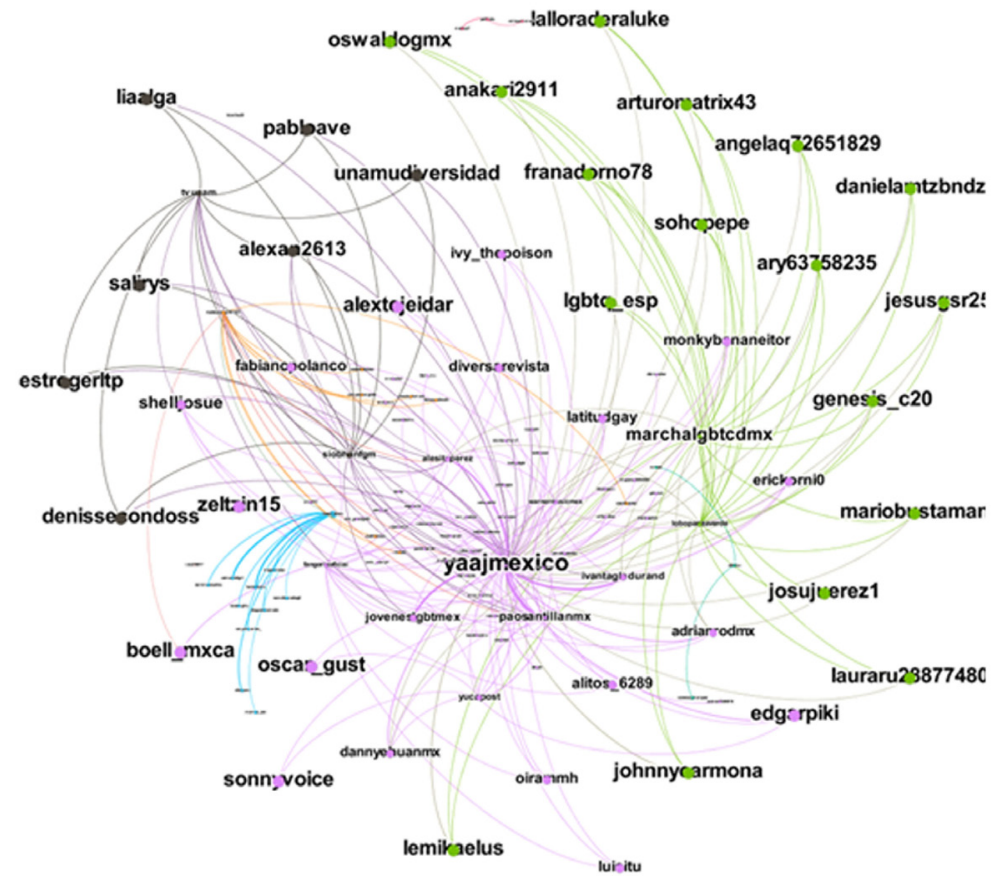

Fuente: Elaboración propia con datos de NodeXL y Gephi.

Esta red muestra la dinámica relacional a través del contenido discursivo y audiovisual que se desarrolla alrededor de dicha etiqueta. A nivel de nodo, los tres perfiles con más vínculos son: Yaaj México (93 vínculos), marchalgbtcdmx (24 vínculos), paosantillanmx (24 vínculos). El primer nodo es de una Asociación Civil, la segunda cuenta 
es un perfil que se dedica a generar y difundir contenido LGBT+ y el tercer nodo es el perfil de una activista.

De manera paralela, el color de los enlaces atiende a las comunidades virtuales que se forman a partir de la interacción. Con esto se pueden identificar diferentes grupos que interactúan mediante el hashtag; de ellos, los vínculos de color morado son aquellos que conforman el grupo más amplio dentro de esta red; en él es posible reconocer tanto a Yaaj México como a Paosantillanmx (marchalgbtcdmx está en la comunidad verde). De la comunidad en color morado, el perfil de Yaaj México puede ser considerado como un hub (Barabási, 2011), esto es, un nodo que posee una posición privilegiada dada la cantidad de vínculos dentro de la red. Un hub puede jugar un doble papel dentro de la estructura, ya que tiene la capacidad de difundir o restringir el flujo de información por los vínculos que posee y de acuerdo con sus intereses.

La asociación civil es integrante del movimiento LGBT+ y sus acciones de intervención en el espacio público híbrido contribuyen a difundir y ampliar el alcance de sus redes y contenido para materializar su agenda. Así, es posible reconocer que esta organización tiene un carácter amplificador del contenido como consecuencia de su activismo, de los trabajos de incidencia que realiza y de su capacidad de intervención en el espacio público.

Para reconocer la relevancia de los integrantes, se clasificaron los 96 perfiles de acuerdo con su vinculación con el movimiento LGBT+ mexicano, por lo que se establecieron las siguientes categorías: activistas, población LGBT+, organizaciones civiles, medios de comunicación digitales independientes (MCDI), simpatizantes del movimiento y perfiles que por la poca información pública no se pudieron reconocer en alguna de las otras categorías. Cada una de estas categorías son importantes dado que, en conjunto, representan los diferentes sectores que integran el movimiento LGBT+. A partir de lo anterior, la Tabla 1 muestra esta distribución. 
Tabla 1

Distribución de perfiles de acuerdo con su rol

\begin{tabular}{|c|c|c|}
\hline Categoría $^{5}$ & $\begin{array}{l}\text { Número } \\
\text { de nodos }\end{array}$ & Definición \\
\hline Activistas & 11 & $\begin{array}{l}\text { Persona con capacidad de interpelación a un grupo } \\
\text { social específico. Esta capacidad deriva de la visibilidad y } \\
\text { reconocimiento de sus acciones en el espacio público, las } \\
\text { cuales cuestionan el estatus quo y promueven el cambio a } \\
\text { favor de la comunidad que representan o de la que forma } \\
\text { parte. }\end{array}$ \\
\hline $\begin{array}{l}\text { Comunidad } \\
\text { LGBT+ }\end{array}$ & 37 & $\begin{array}{l}\text { Conjunto de personas que se reconocen como parte de una } \\
\text { comunidad, ya que comparten rasgos de diversa índole } \\
\text { presentes en sus integrantes, pero también esos rasgos los } \\
\text { diferencian de otros grupos sociales; esto puede contribuir } \\
\text { a su inclusión y/o exclusión. El miembro de una comunidad } \\
\text { tiene una posibilidad contingente de activismo dentro de } \\
\text { su grupo: puede convertirse en una persona activista o } \\
\text { integrarse a una organización social que promueve acciones } \\
\text { en favor de dicha comunidad. En algunos casos puede ser } \\
\text { ambas o solo realizar acciones de activismo soft. }\end{array}$ \\
\hline $\begin{array}{l}\text { Organización } \\
\text { civil }\end{array}$ & 4 & $\begin{array}{l}\text { Colectivo legítima y/o legalmente constituido que realiza } \\
\text { trabajo de incidencia en diferentes ámbitos a favor del grupo } \\
\text { social al que pretenden ayudar. Sus integrantes pueden o no } \\
\text { ser miembros del grupo social al que enfocan sus acciones } \\
\text { Los trabajos de incidencia de una organización civil se } \\
\text { realizan dentro de la comunidad a través de la construcción } \\
\text { de espacios seguros o el fortalecimiento del sentido de } \\
\text { comunidad, y fuera de ella mediante la búsqueda de aliados } \\
\text { estratégicos y el empuje de las demandas que constituyen } \\
\text { su agenda. }\end{array}$ \\
\hline $\begin{array}{l}\text { Medios de } \\
\text { comunica- } \\
\text { ción digital } \\
\text { independien- } \\
\text { tes (MCDI) }\end{array}$ & 8 & $\begin{array}{l}\text { Proyectos digitales enfocados en la producción, distribución } \\
\text { y consumo de información de interés para un grupo social. E } \\
\text { contenido que generan y difunden satisface dos necesidades } \\
\text { para la comunidad en cuestión: una de carácter informativo } \\
\text { ante la falta de contenido en medios de comunicación } \\
\text { tradicionales; y otro de carácter comunicativo, al articular } \\
\text { un discurso que cuestiona las representaciones sociales y } \\
\text { los imaginarios colectivos que contribuyen al estigma de } \\
\text { dicha comunidad. Los costos económicos de estos proyectos } \\
\text { son bajos y los alcances de visibilidad son amplios dada la } \\
\text { estructura reticular de Internet. }\end{array}$ \\
\hline
\end{tabular}

5 La clasificación es arbitraria dada la complejidad de los perfiles y la disponibilidad de la información. En este sentido, vale la pena mencionar que en la categoría de simpatizante se agruparon perfiles de instituciones gubernamentales y universitarias, dado que por el contexto de visibilidad y movilidad en junio, estos organismos tienden a realizar acciones visibles de reconocimiento hacia las comunidades LGBT+ en México. 


\begin{tabular}{lll}
\hline Categoría $^{5}$ & $\begin{array}{c}\text { Número } \\
\text { de nodos }\end{array}$ & Definición \\
\hline Simpatizante & 24 & $\begin{array}{l}\text { Persona, institución, organización civil o empresa que } \\
\text { no se adscribe como miembro de una comunidad, pero } \\
\text { concuerda con las demandas y/o reconoce la desigualdad } \\
\text { que viven los miembros de dicho grupo social. Su relación } \\
\text { es de apoyo y la construcción de este tipo de relación es de } \\
\text { diferente índole: puede tener un interés económico, político } \\
\text { cultural o poseer un vínculo con uno o varios miembros } \\
\text { de esa comunidad. También se les denomina aliados, } \\
\text { porque su apoyo contribuye a una mayor visibilidad de las } \\
\text { demandas y un mayor cuestionamiento al estatus quo. }\end{array}$ \\
\hline Sin Datos & 10 & No aplica. \\
\hline
\end{tabular}

Si se reconoce el papel de cada nodo dentro de este proceso, será posible renombrar la estructura reticular para observar cómo es que los vínculos se desarrollan no solo entre los activistas y organizaciones que promueven cambios a favor de la comunidad LGBT+, sino que los demás miembros LGBT+ están presentes por la dinámica relacional que adquieren mediante sus perfiles en Twitter. La Figura 3 muestra la misma distribución de los nodos con este nuevo valor a partir del rol.

Figura 3

Red de interacción a partir del rol

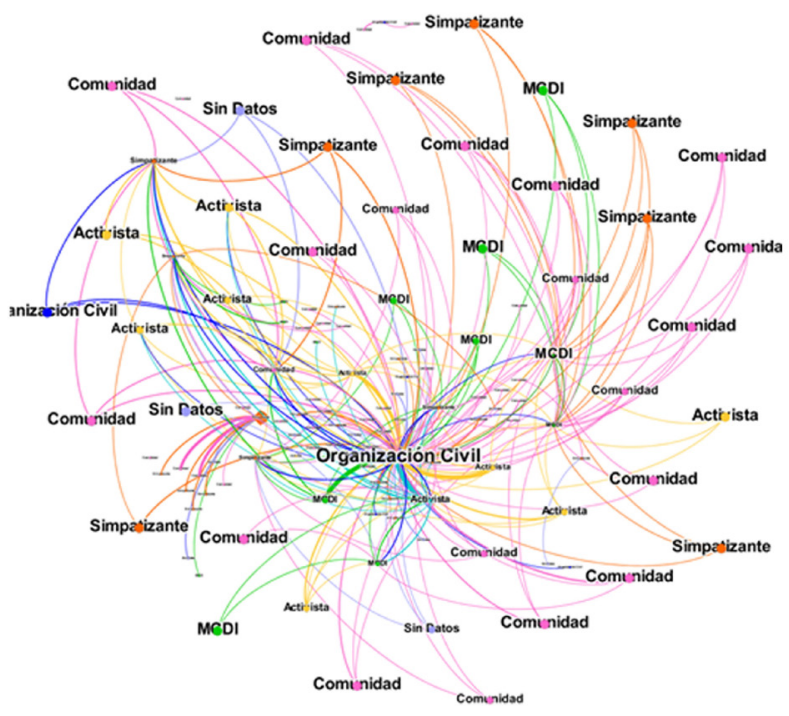


Cada color representa el tipo de rol que posee el nodo que utilizó o compartió el hashtag. Así es posible observar una pluralidad de nodos, cuyos roles y funciones tienen un carácter conectivo: los integrantes de la comunicación, los MCDI y activistas utilizan esta etiqueta para hacer conversaciones y ello, a su vez, genera más vínculos que terminan en una red cuyo contorno está determinado por su propia dinámica orgánica.

Por tanto, estos roles permiten evidenciar el carácter reticular de este movimiento, donde no existen jerarquías o procesos direccionales que restrinjan los flujos de interacción social (Olmedo, 2020). De esta manera, el movimiento LGBT+ mexicano reafirma su carácter reticular, lo que le permite avanzar en la materialización de su agenda.

Cabe mencionar que los vínculos que construyen la red pueden o no descansar en procesos previos de interacción entre los perfiles, es decir, que exista ya una vinculación previa entre los usuarios que allí se presentan. Lo anterior daría paso a considerar que estos flujos de información pueden, por una parte, utilizar los canales desarrollados entre los perfiles como consecuencia de procesos de interacción anteriores, o bien, establecer potenciales vínculos derivados de la interacción de los perfiles con el contenido.

A nivel de flujos de información destacan dos elementos a considerar dentro de las redes que se forman en Twitter: uno es el contenido y otro el sentido simbólico del contenido. Sobre el primero se puede identificar en la Figura 4 que en esta red predominó como detonante de los vínculos.

Figura 4

Cartel oficial de 43 Marcha del Orgullo. Ciudad de México

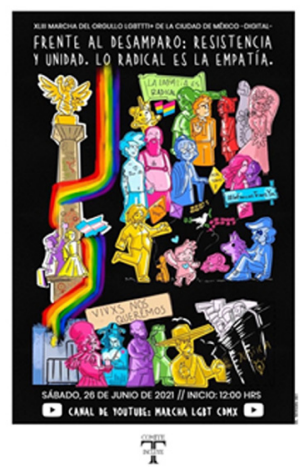

Fuente: Cervantes (2021). 
El cartel oficial de la marcha en la Ciudad de México fue parte del contenido audiovisual que abrió paso a la interacción: compartir, comentar, seguir o reaccionar a este contenido establece el vínculo entre quien lo difunde y quien reacciona a él. La relevancia de este cartel como contenido con mayor difusión es, por un lado, su histórico papel dentro de la organización y difusión de la marcha del Orgullo en la Ciudad de México, así como por la información que ofrece: el número de la marcha que se realiza, la fecha y hora en que se desarrollará, la demanda a la que se enfoca la marcha y, en este caso, dadas las condiciones de pandemia, el canal de YouTube en donde se difundieron las actividades de visibilidad e (inter)acción.

En el nivel simbólico, esta red no solo estuvo desarrollada a partir del hashtag \#Orgullo2021Mx, sino que también se incluyeron otras etiquetas, las cuales evidencian de una u otra forma el desarrollo de un significado compartido a partir de estas herramientas de comunicación. En la Tabla 2 se muestra los hashtags más utilizados dentro de esta red.

Tabla 2

Etiquetas utilizadas dentro de la red

\begin{tabular}{lc}
\hline \multicolumn{1}{c}{ Etiqueta } & Número de veces empleada en la red \\
\hline \#YaajRecomienda & 222 \\
\hline \#Orgullo & 134 \\
\hline \#LGBTI & 64 \\
\hline \#LoveIsLove & 55 \\
\hline \#NadaQueCurar & 36 \\
\hline \#PrideMonth & 14 \\
\hline
\end{tabular}

Fuente: Elaboración propia con datos de NodeXL.

Estas otras etiquetas son parte de la dinámica relacional que yace en Twitter y a la par son el resultado de la apropiación de la tecnología por parte de los usuarios. Su construcción debe atender tanto las restricciones de las posibilidades prestablecidas por la plataforma sociodigital como el potencial valor simbólico que le permita ser replicado por aquellas personas que reconocen dicho código discursivo.

Finalmente, cobra relevancia la cantidad de elementos que pueden ser obtenidos de manera adicional a las vinculaciones que se desarro- 
llan en la red, en este caso emanada de un hashtag. Esto no es más que producto de la dinámica relacional a la que cada perfil se adhiere de manera consciente e inconscientemente en el espacio digital; por ello, resulta relevante observar no solo la vinculación de los datos, sino su uso para entender las formas en que la comunicación y la información confluyen en procesos específicos que se desarrollan en Internet.

\section{Discusión y conclusiones}

La pandemia del coronavirus ha permitido redimensionar las acción de los movimientos sociales en el espacio público. A partir de lo observado en este trabajo se ha podido delimitar, visualizar y analizar la dinámica relacional detrás de una etiqueta creada y utilizada por una parte del movimiento LGBT+ mexicano en este contexto de incertidumbre.

Las preguntas que motivaron esta investigación y la hipótesis para responderlas se materializan en las estructuras reticulares que se forman mediante la interacción de los usuarios entre sí y con el contenido. El hashtag, y el contenido que lo acompaña, permite interpelar a un tipo de usuarios de Twitter; esos usuarios/perfiles pueden responder de manera positiva o negativa a dicho contenido o etiqueta, por lo que aquella interacción resultante con el contenido y los usuarios que lo difunden responde al posicionamiento del individuo mediante su perfil como lugar de enunciación. De esta manera:

Las redes sociodigitales se constituyen como una extensión del individuo que las crea y modela según sus intereses comunicativos, informativos e identitarios; ellas se vuelven un soporte más donde el sujeto (sea individual o colectivo) manifiesta elementos propios específicos de su ser que de manera arbitraria selecciona, extrapola y adjetiva como cualidades sui generis a su condición. (Olmedo, 2020, pp. 224-225).

Así, los perfiles se vuelven un espacio no solo desde el cual se interactúa en Internet, sino un lugar desde el que se posiciona el sujeto ante lo que allí acontece. El perfil es el nodo desde el cual se es/está en el mundo digital, por lo que desde ese lugar de enunciación se desarrollan prácticas comunicativas que diseñan el perfil, la identidad y el posicionamiento ante temas de interés para quien opera esa cuenta. 
Por otro lado, con los resultados obtenidos es posible reconocer que actualmente este movimiento está consolidando su experiencia alrededor del uso que puede darle a Internet y las plataformas sociodigitales como Twitter. Las redes que se han presentado muestran diferentes características de un proceso de interacción social que se da en los espacios digitales; estos vínculos no solo dependen del valor tecnológico, sino que pueden estar mediados por aquellos enlaces que el individuo genera en su biografía, mismos que puede replicar parcial o totalmente. Este uso constante en la forma de socializar permite abrir nuevos espacios para la investigación y para la intervención en un espacio público que hoy ya no es exclusivamente digital o no digital, sino una confluencia de ambos.

Futuras investigaciones pueden ahondar en los efectos desencadenados en las redes sociales, digitales y sociodigitales que posee un perfil cuando comparte este tipo de contenido. Sin duda este posicionamiento y capacidad de control está en permanente negociación con los usuarios con los que se interactúa. De esta manera, reconocer el impacto sobre las redes resulta medular para profundizar en las formas en que estas acciones de activismo soft permiten hablar de identidades y preferencias sexuales que viven el estigma derivado del heterosexismo.

La vinculación entre activistas, organizaciones, comunidad, proyectos mediáticos y las personas simpatizantes de este movimiento social muestra la confluencia de un grupo que actúa en red y a través de la red. La posibilidad de establecer una comunicación e interacción deslocalizada abre camino para reducir los costos de desplazamiento y los tiempos del territorio; futuras investigaciones pueden abonar a las ventajas y retos que surgen de la relación entre comunicación y espacio-tiempo en las plataformas sociodigitales.

De los sectores que integran esta comunidad, cobra relevancia lo que en este trabajo se han nombrado medios de comunicación digitales independientes (MCDI). Su relevancia radica en su función informativa y de alcance dado que se especializan en la producción y difusión de contenido que los medios de comunicación tradicionales no ofrecen; el bajo costo de mantenimiento de un perfil y los nulos procesos de gestión ante el Estado hacen que las plataformas sociodigitales se conviertan en canales factibles para construir la resistencia comunicativa y relacional. Las formas en que medios como Mar- 
chalgbtcdmx, Escándala, Soy Homosensual y Seis Franjas México se consolidan entre la comunidad LGBT+ mexicana y latinoamericana adquiere relevancia para reconocer su capacidad de organización, los retos de su gestión y los alcances de su propia función orgánica dentro de este movimiento social contemporáneo (Pérez, 2018).

Todas estas experiencias y formas de interacción se acumulan para configurar una experiencia derivada de la apropiación; su desarrollo se manifiesta colectivamente cada vez que se vinculan más personas con estas acciones conectivas. Este conocimiento adquiere valor no solo por su aplicación en un contexto mediatizado por la tecnología, sino porque ese nuevo saber es resultado de la constante actualización tecnológica de los integrantes del movimiento LGBT+ y la participación de las juventudes LGBT+ que entienden las implicaciones del uso de Internet en la vida cotidiana.

Esta relación dialógica-dialéctica entre diferentes generaciones es un factor que influye en lo que en este trabajo se ha propuesto definir como sociedad beta: son las nuevas generaciones quienes abonarán a comprender y aprehender estos potenciales usos para incrementar la visibilidad y, con ello, tejer la contrahegemonía a nivel comunicativo, social y simbólico. Son las juventudes LGBT+ quienes consolidarán el giro comunicacional en Internet mediante la integración y afinación de su uso para despojarse del estigma y reivindicar su posición en el mundo contemporáneo.

La apropiación de Internet y las plataformas sociodigitales que realiza en su conjunto el movimiento LGBT+ mexicano se suma a la trayectoria histórica de iniciativas desarrolladas para apropiarse de los medios de comunicación. Cuestionar los discursos y prácticas de discriminación y estigmatización que encuentran eco en la sociedad, en los medios de comunicación masiva y también en Internet sigue siendo una premisa fundamental para esta acción colectiva contemporánea. Con Internet, los grupos históricamente discriminados pueden gestionar canales para desmantelar el estigma y construir un discurso alternativo emanado de su horizonte de posibilidades. De allí que el análisis de la etiqueta resulta importante no solo porque se consolida como un elemento multipropósito de tal valor que, según Feixa et al. (2016), será el carácter más representativo de las juventudes contemporáneas. 
De hecho, en términos conceptuales el hashtag tiene un potencial semántico y relacional que no ha sido analizado suficientemente. A nivel semántico, La Rocca (2020) menciona que el hashtag "va más allá de la etiqueta en sí y se abre a posibles mundos de significación que necesitan una especificación, porque solo si entendemos qué es cada hashtag, podremos identificar sus efectos en la construcción de la realidad social" (p. 48). Por ello, el análisis de las etiquetas a nivel de red debe considerar inexorablemente el contexto de aparición, la construcción simbólica que se da con su difusión y el declive que presenta cuando la acción convergente a la que refiere termina, sea esta una reunión, un evento o, como en este caso, el mes del Orgullo en México.

A nivel relacional los hashtags se convierten en productos que tienen la capacidad de funcionar como rastreadores digitales; el uso e interacción que se hace de ellos permite investigar los perfiles, la cantidad de interacciones, así como otros datos tales como la fecha, la hora, la ubicación y el contenido que acompaña dicha etiqueta. Reguillo (2017) insiste que los hashtags tienen la posibilidad de posicionar el perfil dentro de la construcción de la opinión pública digital; pero a partir de lo analizado es factible reconocer que la creación, difusión e interacción con el contenido y los hashtags implica un posicionamiento alrededor del discurso que acompaña dichos elementos.

Por tanto, los hashtags también encarnan un posicionamiento político, simbólico y comunicativo alrededor de los significados de dichas etiquetas. De allí que contextualizar su surgimiento abona a reconocer quiénes lo publican, lo difunden y por qué. En el caso analizado, quienes más participaron en su difusión y creación fueron tanto los integrantes de la comunidad LGBT+ que no se autorreconocen como activistas, por un lado, y aquellas personas que simpatizan con las demandas y acciones de este movimiento social.

La creación y difusión de las etiquetas encarnan en buena medida el repertorio de acción conectiva (Reguillo, 2017): son estos recursos digitales con los que se interactúa de manera constante lo que abre la posibilidad a reconocerlos como potenciales herramientas de difusión que permiten generar conversación alrededor de un tema. Estas estructuras se configuran a través de la innovación y apropiación de dicha tecnología por parte del usuario, por lo que su codificación define un lugar de enunciación desde el cual se habla sobre un tema. Así, 
su función como etiqueta permite, además, establecer relaciones con imágenes, links, sonidos y videos que posibilitan que su construcción adquiera un significado en quienes deciden reaccionar y retuitear dicho hashtag. En otras palabras, la construcción del sentido no se da exclusivamente por el individuo que crea la etiqueta, sino a través del uso que le dan las personas que lo replican y comentan en Twitter.

La consolidación de Yaaj México como un hub deriva en buena medida de los trabajos de incidencia que realiza en la Ciudad de México, los canales políticos que ha establecido para empujar demandas como la legislación en contra de las terapias de conversión, y la ampliación de sus redes de interacción y comunicación mediante sus perfiles. Reconocer los hubs dentro de la red es medular, ya que así es posible identificar las razones que posicionan dicho nodo con ese atributo. En términos generales, la trayectoria de activismo y vinculación político/ mediática de esta organización la vuelven un ejemplo de cómo es posible construir redes en red y ampliar las ya existentes mediante sus cuentas en diferentes plataformas sociodigitales.

Finalmente, en este ejercicio analítico se reafirma el carácter comunicativo que exponen de manera estratégica los movimientos sociales contemporáneos como el LGBT+. Otras investigaciones deberán profundizar en los usos de estos recursos tecnosociales y en las formas de visualizarlos o analizarlos de tal manera que contribuyan a desentramar los procesos de socialización que desarrollan los movimientos sociales contemporáneos en la sociedad beta.

\section{Referencias}

Albury, K. (2018). Sexual expression in social media. En J. Burgess, A. Marwick, y T. Poell (Eds.), The SAGE Handbook of social media (pp. 444-462). SAGE Publications.

Barabási, A.-L. (2011). Introduction and keynote to a networked self. En Z. Papacharissi (Ed.), A networked self (pp. 1-14). Routledge.

Barranquero, A. (2012). Redes digitales y movilización colectiva. Del 15-M a nuevas prácticas de empoderamiento y desarrollo local. En M. Martínez y F. Sierra Caballero (Coords.), Comunicación y desarrollo. Prácticas comunicativas y empoderamiento local (pp. 377-400). Gedisa. 
Bucher, T. y Heldmond, A. (2018). The affordances of social media platforms. En J. Burgess, A. Marwick, y T. Poell (Eds.), The SAGE Handbook of social media (pp. 233-253). SAGE Publications.

Burgos, E. K. (2015). La tecnopolítica y la acción colectiva en la sociedad red. Razón y Palabra, 19(89), 1-22. https://revistarazonypalabra.org/index.php/ryp/article/view/260

Candón-Mena, J. (2019). Movimientos conectados: Abordajes tecnopolíticos. IC-Revista Científica de Información y Comunicación, (16), 23-35. https://icjournal-ojs.org/index.php/ IC-Journal/article/view/525/396

Candón-Mena, J. (2011). Internet en movimiento: nuevos movimientos sociales y nuevos medios en la sociedad de la información [Tesis doctoral publicada]. Universidad Complutense de Madrid.

Castillo, M. (4 de octubre de 2016). Luis González de Alba escribió el Primer Manifiesto en Defensa de los Homosexuales. Revista Siempre. http://www.siempre.mx/2016/10/luis-gonzalezde-alba-escribio-el-primer-manifiesto-en-defensa-de-loshomosexuales/

Cervantes, A. (28 de mayo de 2021). Conoce el cartel oficial de la Marcha del Orgullo LGBTTTI de la CDMX2021. TimeOut. https:// www.timeoutmexico.mx/ciudad-de-mexico/gay-y-lesbico/ conoce-el-cartel-oficial-de-la-marcha-del-orgullo-lgbttti-dela-cdmx-2021

Ciszek, E.(2017). Todo mejora en el ambiente:An analysis of digitalLGBT activism in Mexico. Journal of Communication Inquiry, 41(4), 313-330. https://doi.org/10.1177\%2F0196859917712980

Craig, R.T.(1999).Communication theoryasafield. Communication Theory, 9(2), 119-161. https://doi.org/10.1111/j.1468-2885.1999. tb00355.x

Deleuze, G. y Guattari, F. (2010). Mil mesetas. Capitalismo y esquizofrenia. Pre-Textos.

Díez, J. (2011). La trayectoria del movimiento Lésbico-Gay en México. Estudios Sociológicos, 29(86), 687-712. https://doi. org/10.24201/es.2011v29n86.237

Díez, J. (2018). La política del matrimonio gay en América Latina. Fondo de Cultura Económica. 
Enguix, B. (2016). Activismo y prácticas digitales en la construcción de una esfera LGBT en España. Dados. Revista de Ciências Sociais, 59(3), 755-787. https://doi. org/10.1590/00115258201691

Feixa, C., Fernández-Planells, A., y Figueras-Maz, M. (2016). Generación hashtag. Los movimientos juveniles en la era de la web social. Revista Latinoamericana de Ciencias Sociales, Niñez y Juventud, 14(1), 107-120. http://www.scielo.org.co/pdf/rlcs/ v14n1/v14n1a07.pdf

Flores-Márquez, D. (2017). Movimientos sociales e Internet en México. En G. Pleyers y M. Garza Zepeda (Coords.), México en movimientos. Resistencias y alternativas (pp. 129-126). Universidad Autónoma de Ciudad Juárez, UACJ-M. A. Porrúa.

Fuchs, C. (2015). Culture and economy in the age of social media. Routledge.

Fuchs, C. (2021). Communication and capitalism. A critical theory. University of Westminster Press.

García, C. y Olmedo, R. A. (2019). El nuevo opio del pueblo: apuntes desde la economía política de la comunicación para (des) entender la esfera digital. Iberoamérica Social: Revista-Red de Estudios Sociales, 7(12), 84-96. https://iberoamericasocial.com/ojs/index.php/IS/article/view/366

González, L. J. y Servín, A. (2017). Métodos cualitativos digitales: un acercamiento a la antropología digital y otras posturas de investigación online. Virtualis, 7(15), 61-80. https://www. revistavirtualis.mx/index.php/virtualis/article/down$\operatorname{load} / 220 / 202$

Gunter, B. (2014). Los procedimientos de las investigaciones cuantitativas. En K. B. Jensen (Ed.), La comunicación y los medios (pp. 379-424). Fondo de Cultura Económica.

Han, B.-C. (2018). Psicopolítica. Herder.

Irys, S. (2018). ¿Qué me cuentas a mí, que sé tu historia? En M. Schuessler y M. Capistrán (Coords.), México se escribe con J. Una historia de la cultura gay (pp. 407-418). DeBolsillo.

La Rocca, G. (2020). La fuerza de un signo. Perspectivas teóricas para el análisis de los hashtags \#. BARATARIA, Revista Castellano-Manchega de Ciencias Sociales, (27), 46-61. https://doi. org/10.20932/barataria.v0i27.559 
Lizárraga, X. (2018). Un devenir de visibilidad y voces. En M. Schuessler y M. Capistrán (Coords.), México se escribe con J. Una historia de la cultura gay (pp. 345-370). DeBolsillo.

Martel, F. (2013). Global gay. Cómo la revolución gay está cambiando el mundo. Taurus.

Mattoni, A. y Treré, E. (2014). Media practices, mediation processes and mediatization in the study of social movements. Communication Theory, 24(3), 252-271. https://doi. org/10.1111/comt.12038

McAdam, D., Tarrow, S., y Tilly, C. (2004). Dynamics of contention. Cambrigde University Press.

Medina, J. A. (2015). Representación social de los homosexuales en los medios de comunicación: devenir, estigmas y la lucha por la igualdad. Universidad Autónoma de la Ciudad de México.

Melucci, A. (2010). Acción colectiva, vida cotidiana y democracia. El Colegio de México.

Olmedo, R. A. (2019). Los medios en la inclusión de la diversidad sexual en la Ciudad de México. Revista Internacional de Ciencias Sociales Interdisciplinarias, 7(2), 187-200. https://doi. org/10.18848/2474-6029/CGP/v07i02/187-200

Olmedo, R. A. (2020). La comunicación emergente en México (20092016): las organizaciones civiles en la lucha por el matrimonio igualitario [Tesis de maestría]. Universidad Nacional Autónoma de México, UNAM. http://132.248.9.195/ptd2020/ agosto/0802920/Index.html

Olmedo, R. A. (2021). Cartografías conectivas: un acercamiento a la construcción de redes sociodigitales del movimiento LGBT+. Chasqui. Revista Latinoamericana de Comunicación, (147), 123-142. https://doi.org/10.16921/chasqui. vlil47.4456

Pérez, D. (2018). Imparables. Radiografía de organizaciones, medios de comunicación y estado de los derechos de las comunidad LGBTI+ en América Latina. Distintas Latitudes.

Pisani, F. y Piotet, D. (2009). La alquimia de las multitudes. Paidós.

Ramón, M. (23 de febrero de 2021). Uso de redes sociales en México: 100 millones acceden al social media, la mayoría a Facebook. Marketing Ecommerce. https://marketing4ecommerce.mx/ uso-de-redes-sociales-en-mexico/ 
Reguillo, R. (2017). Paisajes insurrectos. Instituto Tecnológico y de Estudios Superiores de Occidente, ITESO.

Ripstein, A. (Dir.). (1977). El lugar sin límites [Película]. YouTube. https://www.youtube.com/watch?v=FE9168dokOQ

Rodríguez, C. A. (2020). La expansión de lo público. Indagaciones tecnopolíticas de la sociedad hiperconectada. Universidad Autónoma Metropolitana-Unidad Cuajimalpa.

Romero, A. (2014). La utopía postfeminismo. Del ciberfeminismo al tecnofeminismo. Cuadernos del Ateneo, (32), 156-169. https://dialnet.unirioja.es/descarga/articulo/5241123.pdf

Rovira, G. (2012). Movimientos sociales y comunicación: la red como paradigma. Anàlisi: quaderns de comunicació i cultura, (45), 91-104. https://raco.cat/index.php/Analisi/article/ view/258164/345470

Sierra, F. y Montero, D. (Coords.) (2016). Videoactivismo y movimientos sociales. Teoría y praxis de las multitudes conectadas. Gedisa.

Simonetto, P. (2017). Movimiento de liberación homosexual en América Latina. Aportes historiográficas desde una perspectiva comparada entre Argentina, Brasil, Chile, Colombia y México (1967-1982). Iberoamericana, 17(65), 157-177. https:// journals.iai.spk-berlin.de/index.php/iberoamericana/article/view/2096/1950

Sola-Morales, S. y Sabariego, J. (2020). Tecnopolítica, recientes movimientos sociales globales e Internet. Una década de protestas ciudadanas. Teknokultura. Revista de Cultura Digital y Movimientos Sociales, 17(2), 195-208. https://doi.org/10.5209/ tekn.66241

Tilly, C. y Wood, L. J. (2010). Los movimientos sociales, 1768-2008. Crítica.

Touraine, A. (2005). Un nuevo paradigma para entender el mundo de hoy. Paidós.

Treré, E. (2020). Activismo mediático híbrido. Friedrich Ebert Stiftung. Wajcman, J. (2006). El tecnofeminismo. Cátedra.

Zallo, R. (2016). Tendencias en comunicación. Gedisa.

Zapata, L. (1979). El vampiro de la colonia Roma. Grijalbo. 